\title{
Introduction and rationale
}

Non-adherence to direct oral anticoagulants (DOACs) remains a matter of concern. Studies using either self-reporting [1-4] or prescription claims data [5,6] to assess adherence have yielded discrepant results as to the extent of non-adherence and have failed to identify concrete predictors. Although electronic monitoring is the most accurate assessment method [7], electronic data on adherence to DOACs are scarce [9]. Furthermore, as evidence emerges that non-adherence to DOACs increases the risk for mortality, stroke and bleeding $[2,6]$, the need to develop effective adherence-improving strategies is being increasingly recognized, but the evidence remains poor [8,9]. Patients with a recent stroke are at a high risk for both recurrence [10] and non-adherence due to neurological and cognitive deficits [11]. Such patients would therefore benefit the most from adherence-improving interventions, but no studies so far have used alarm-delivering multicompartment medication systems to improve the adherence of DOAC-treated stroke patients. We aim to use electronic monitoring and a reminder-delivering medication system in a cohort of polymedicated patients with a recent stroke in order to:

- accurately measure non-adherence to DOACs and thus to identify predictors and valid self-reporting strategies and evaluate its association with clinical outcomes - assess the adherence-improving effect of an educational and reminder-based intervention in DOAC-treated patients after a recent stroke.

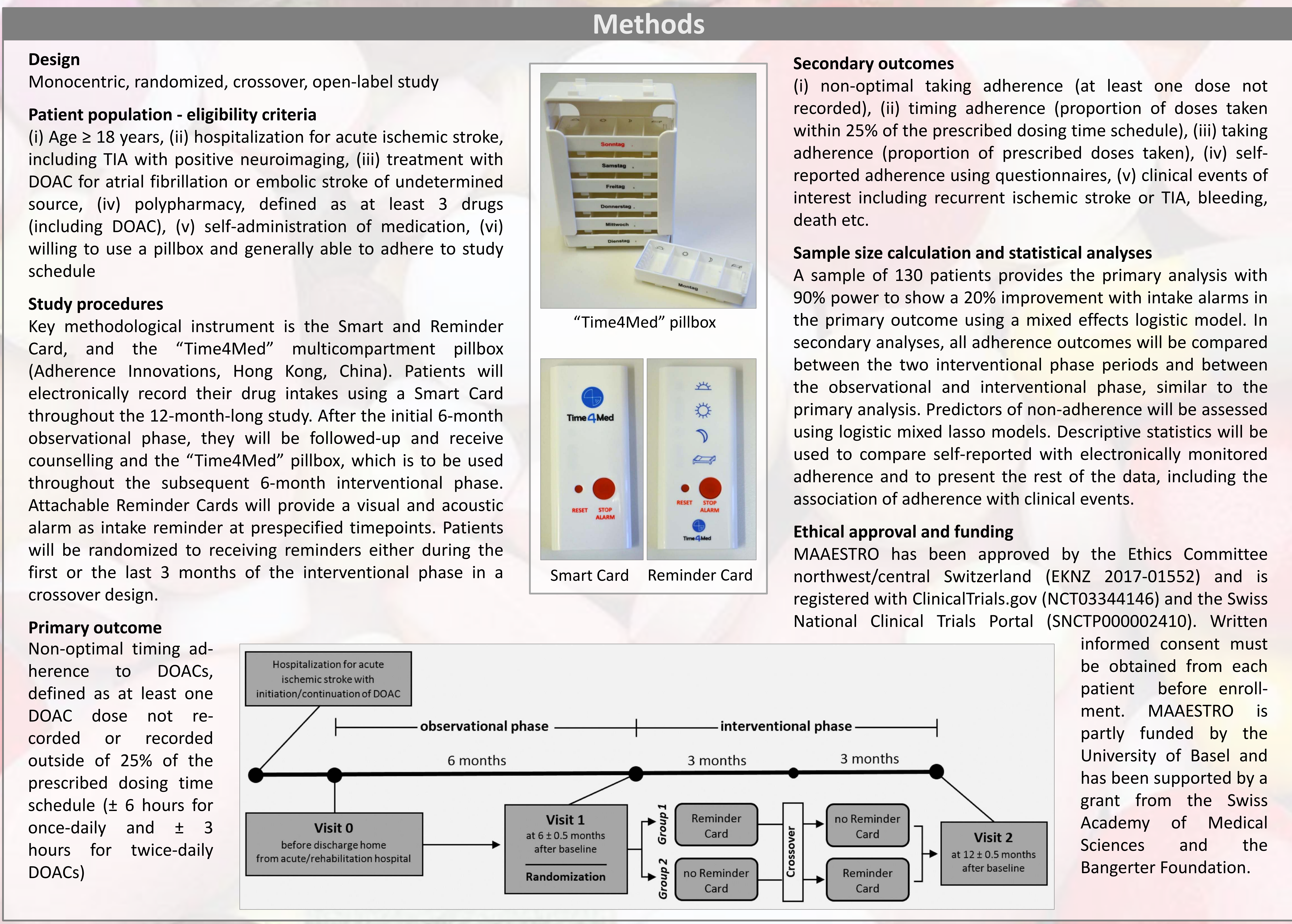

\section{Conclusion}

MAAESTRO will investigate aspects of non-adherence using electronic monitoring and evaluate the effect of an educational, reminder-based, adherence-improving intervention in the previously unexplored population of DOAC-treated patients with a recent stroke using electronic monitoring. Enrollment is currently ongoing.

\section{References}

1. Luger S, Hohmann C, Niemann D, et al. Adherence to oral anticoagulant therapy in secondary stroke prevention - impact of the novel oral anticoagulants. Patient Prefer Adherence 2015;9:1695-705.

Antion Anticoagulants in Turkey. Clinical and applied thrombosis/hemostasis : official journal of the International Academy of Clinical and Applied 3. Polymeris AA, Traenka C. Hert L, et al. Frequency

Fibrillation in Clinical Practice. European neurology 2016;76(3-4):187-93.

4. Castellucci LA, Shaw J, van der Salm K, et al. Self-reported adherence to anticoagulation and its determinants using the Morisky medication adherence scale. Thromb Res 2015;136(4):727-31.

5. Manzoor BS, Lee TA, Sharp LK, et al. Real-World Adherence and Persistence with Direct Oral Anticoagulants in Adults with Atrial Fibrillation. Pharmacotherapy 2017.
6. Borne RT, O'Donnell C, Turakhia MP, et al. Adherence and outcomes to direct oral anticoagulants among patients with atrial fibrillation findings from the veterans health administration. BMC cardiovascular disorders 2017;17(1):236.

7. Sutton $S$, Kinmonth AL, Hardeman W, et al. Does electronic monitoring influence adherence to medication? Randomized controlled trial 8. Desteghe L, Vijgen J, Koopman P, et al. Telemonitoring-based feedback improves adherence to non-vitamin K antagonist ora anticoasulants intake in patients with atrial fibrillation. European heart journal 2018.

9. Boehme P, Wienand P, Herrmann M, et al. New digital adherence devices could prevent millions of strokes from atrial fibrillation by the end of the next century. Medical hypotheses 2017;108:46-50.

10. The International Stroke Trial (IST): a randomised trial of aspirin, subcutaneous heparin, both, or neither among 19435 patients with acute ischaemic stroke. The Lancet;349(9065):1569-81.

11. Sukainah Al A, Terry Q, William D, et al. Predictive factors of non-adherence to secondary preventative medication after stroke or transient ischaemic attack: A systematic review and meta-analyses. European Stroke Journal 2016;1(2):65-75. 